\title{
Les tuiles médiévales de l'abbaye cistercienne de Quincy (Tanlay, Yonne), 2007-2008
}

\section{Sylvain Aumard}

\section{(2) OpenEdition}

1 Journals

\section{Édition électronique}

URL : https://journals.openedition.org/cem/11309

DOI : 10.4000/cem.11309

ISSN : 1954-3093

Éditeur

Centre d'études médiévales Saint-Germain d'Auxerre

\section{Édition imprimée}

Date de publication : 15 août 2009

Pagination : $97-98$

ISSN : 1623-5770

\section{Référence électronique}

Sylvain Aumard, « Les tuiles médiévales de l'abbaye cistercienne de Quincy (Tanlay, Yonne),

2007-2008 », Bulletin du centre d'études médiévales d'Auxerre / BUCEMA [En ligne], 13 | 2009, mis en ligne le 16 septembre 2009, consulté le 22 septembre 2022. URL : http://journals.openedition.org/cem/

11309 ; DOI : https://doi.org/10.4000/cem.11309

Ce document a été généré automatiquement le 22 septembre 2022.

\section{(c)}

Creative Commons - Attribution - Pas d'Utilisation Commerciale - Partage dans les Mêmes Conditions 4.0 International - CC BY-NC-SA 4.0

https://creativecommons.org/licenses/by-nc-sa/4.0/ 


\title{
Les tuiles médiévales de l'abbaye cistercienne de Quincy (Tanlay, Yonne), 2007-2008
}

\author{
Sylvain Aumard
}

1 Dans le cadre de la rénovation de la couverture du logis de l'abbé (appelé aussi "bâtiment des moines ») de l'abbaye de Quincy, une étude des tuiles médiévales a été demandée par le maître d'ouvrage. Mis en évidence lors de notre intervention en 2004, ces matériaux ont pu bénéficier cette fois-ci d'un contexte d'étude exceptionnel. Alors que sur la plupart des chantiers ils sont systématiquement remplacés et traités comme de simples gravats, ici, une fois déposés, ils étaient à la disposition de l'archéologue durant la réparation de la charpente, avant d'être réutilisés sur le même toit. Le principal atout de cette opération était la possibilité de corriger la typologie à n'importe quel stade de son élaboration et de recueillir un maximum d'indices dimensions, poids, morphologie, techniques. L'accent a porté essentiellement sur la première tranche de restauration - croupe nord de la toiture - dans la mesure où l'étude a rapidement permis d'atteindre un échantillonnage très conséquent de tuiles potentiellement médiévales (environ 400). La seconde a été mise à profit uniquement dans le but de compléter les catégories faiblement représentées.

2 La typologie regroupe principalement des tuiles sans crochet à un trou (type 1, 205 ex.) ou à deux trous (type 2, 120 ex.). Déjà reconnues sur d'autres sites médiévaux de la région, il s'agit des formes les plus faciles à reconnaître du fait de leur système de fixation original. Certaines possédaient encore leur cheville en bois partiellement conservée. Une autre catégorie a focalisé notre attention : il s'agit des tuiles à gros crochet latéral moulé (type 4, 36 ex.), dont la similitude avec l'exemplaire découvert en 2004 dans une des culées des arcsboutants du chevet de Pontigny (fin XIlesiècle) est absolument incontestable. Cette datation devrait bientôt être corroborée par les analyses en thermoluminescence en cours de réalisation sur les 
typologies de la cathédrale d'Auxerre, puis sur celles à venir des corpus de Pontigny et de Vermenton (C2RMF, musée du Louvre) ${ }^{1}$. L'observation de caractères techniques et dimensionnels communs aux productions des types 1,2 et 3 laisse envisager une datation très comparable des types sans crochet : empreinte de tissu laissée lors du moulage, formes très régulières et calibrées par reprise systématique des bords avec un outil tranchant. En revanche, seul le type 4 a parfois reçu une glaçure plombifère variant du vert-noir au noir-marron sur le pureau exposé aux intempéries (12 ex.). Cette particularité fait des tuiles de Quincy un corpus de toute première importance dans l'étude des terres cuites architecturales, car celui-ci conserve vraisemblablement les glaçures parmi les plus anciennes attestées par l'archéologie sur des matériaux de couverture, jusqu'alors évoquée dans la documentation écrite et quelques découvertes ${ }^{2}$. Les futures datations radiocarbones sur deux charbons de bois prélevés au cœur de la pâte ne manqueront pas de nous tenir en haleine... En outre, ces typologies ont également livré des exemplaires dont la morphologie les destinait à des fonctions très spécifiques: formes gironnées pour toitures de tour ou d'abside, formes avec encoche sommitale probablement pour la ventilation (?).

Enfin, les deux autres catégories (type 5 et 6 ) ne peuvent, pour le moment, être datée à cause de la physionomie extrêmement banale de leur crochet retroussé (latéral ou médian).

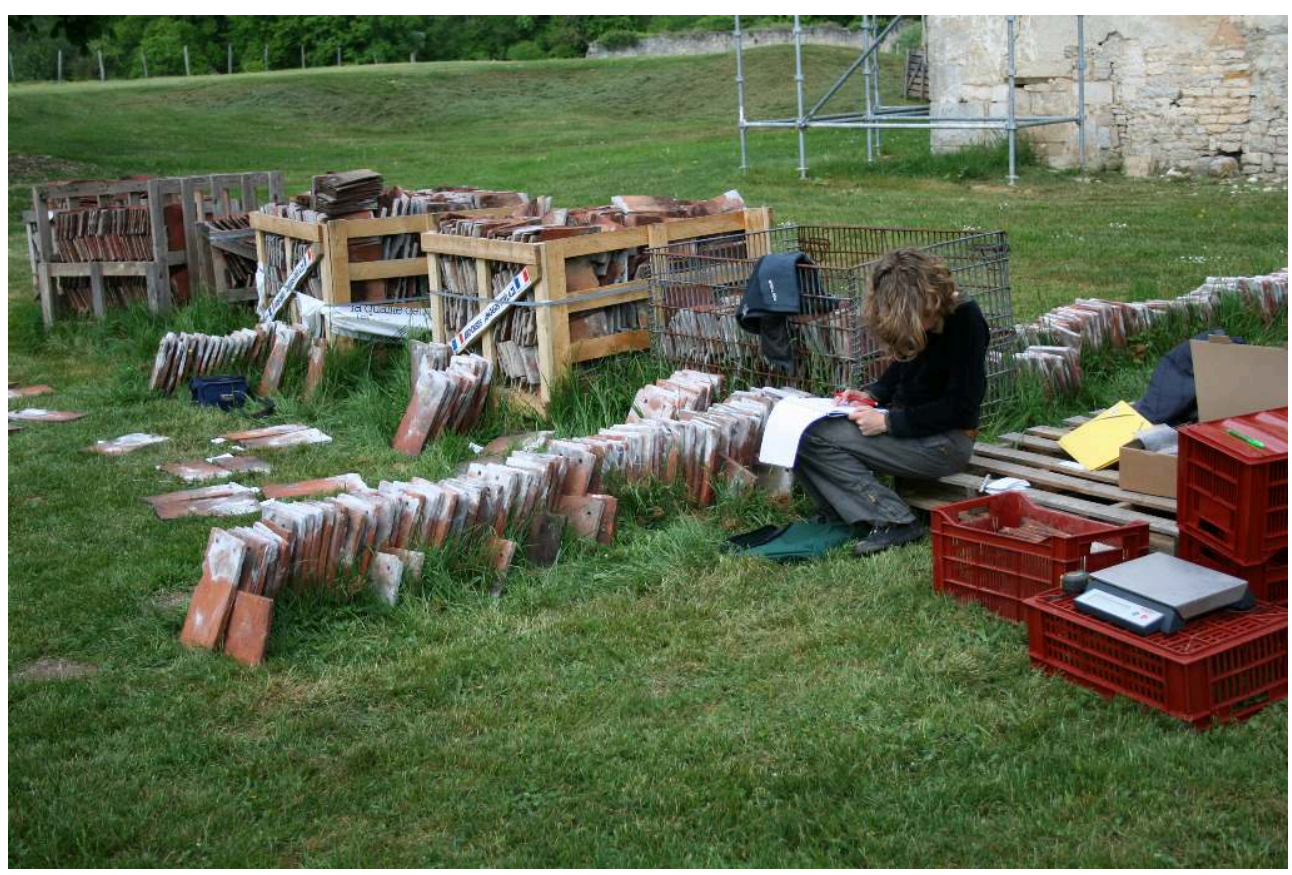




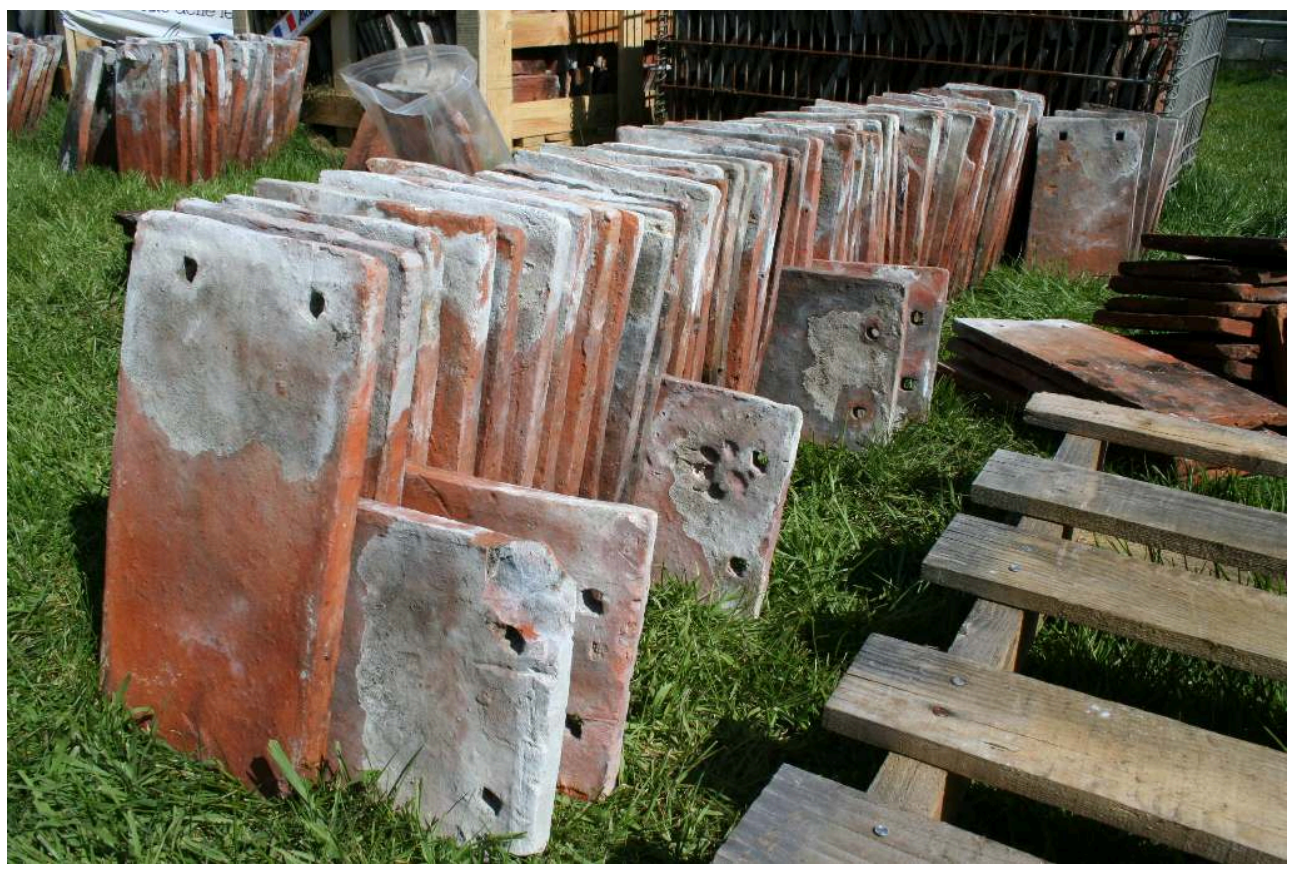

Fig. 1 - Tanlay, abbaye de Quincy : étude de grandes séries de tuiles médiévales lors de la restauration des couvertures du logie de l'abbé (cl. S. Aumard).

\section{NOTES}

1. Analyses réalisées par Antoine Zink et Elisa Porto (C2RMF) dans le cadre du PNRC obtenu au ministère de la Culture (Mission de la recherche et de la technologie): «Les tuiles des monuments de l'Yonne... ", voir l'article dans ce même bulletin.

2. Geste de l'évêque Guillaume de Toucy (1167-1181), rédigée vers 1183 : « II recouvrit toute l'église de nouvelles poutres, fermes et tuiles - dont certaines garnies de plomb..." ", Les gestes des évêques d'Auxerre, M. SOT dir., t. 2, Paris, 2006, p. 128-129 ; F.ÉPAUD, De la charpente romane à la charpente gothique en Normandie. Évolution des techniques et des structures de charpenterie aux XIIe-XIIIe siècles, Caen, 2007, p. 126-127; C. BAUDART, «Étude sur les tuiles mises au jour dans l'unité stratigraphique 1568 », in Château de Brie-Comte-Robert, Rapport de fouille, 2003, inédit, 7 p. (information de l'auteur). 
INDEX

Mots-clés : abbaye, tuile

Index géographique : France/Tanlay-Quincy 